D. Steinbart ${ }^{1}$, J. Bösel ${ }^{2}$, M. Holtkamp ${ }^{1,3}$

${ }^{1}$ Klinik für Neurologie, Campus Virchow-Klinikum, Charité - Universitätsmedizin Berlin, Berlin, Deutschland

${ }^{2}$ Klinik für Neurologie, Klinikum Kassel, Kassel, Deutschland

${ }^{3}$ Epilepsiezentrum Berlin-Brandenburg, Institut für Diagnostik der Epilepsien, Evangelisches Krankenhaus Königin Elisabeth Herzberge, Berlin, Deutschland

\title{
Unterscheidung psychogener nicht-epileptischer und epileptischer Anfälle
}

\section{Erwiderung}

Zum Leserbrief von Rösche J (2021) Nicht immer ist Weinen psychogen. DGNeurologie. https://doi.org/10.1007/s42451-021-00390-y.

\section{Originalbeitrag}

Steinbart D, Bösel J, Holtkamp M (2021) Epileptologische Notfälle. DGNeurologie 4:121-131. https://doi.org/10.1007/s42451-021-003186.

Wir bedanken uns bei Herrn Rösche für den Hinweis, dass in Einzelfällen auch bei epileptischen Anfällen Weinen als Teil der iktalen Anfallssemiologie beobachtet werden kann. Auch in der zitierten Studie [1] hatte der Großteil der Patienten mit iktalem Weinen psychogene nicht-epileptische Anfälle. Bezogen auf die hohe Gesamtzahl der in dieser Studie untersuchten Patienten kann aus den angeführten Daten eine Spezifität des iktalen Weinens für psychogene nicht-epileptische Anfälle von etwa $99 \%$ berechnet werden, dieser Wert liegt sehr nah an der Angabe (100\%) der in unserem Artikel zitierten Studien. Alle 10 von uns aufgeführten klinischen Merkmale haben eine hohe Spezifität von 90-100\% für psychogene nichtepileptische bzw. für epileptische Anfälle, aber kein Merkmal allein beweist das Vorliegen einer der beiden Diagnosen. Diese können - jenseits des Goldstandards Video-Elektroenzephalografie-Aufzeichnung - nur in Zusammenschau aller klinischen Informationen gestellt werden.

Literatur

1. Asadi-Pooya A, Wyeth D, Sperling M (2016) Ictal crying. Epilepsy \& Behavior 59:1-3. https://doi.org/10.1016/j.yebeh.2016.03.012

\section{Korrespondenzadresse}

\section{Dr. D. Steinbart}

Klinik für Neurologie, Campus Virchow-Klinikum Charité - Universitätsmedizin Berlin

Augustenburger Platz 1, 13353 Berlin, Deutschland

david.steinbart@charite.de

Interessenkonflikt. D. Steinbart, J. Bösel und M. Holtkamp geben an, dass kein Interessenkonflikt besteht. 